\title{
Thymic Stromal Lymphopoietin Is Associated With Disease Activity in Systemic Lupus Erythematosus
}

Xiaofei Lai

Chongqing Medical University https://orcid.org/0000-0002-7230-1617

\section{Huiqing Yang}

Chongqing Medical University

\section{Hao Ding}

Chongqing Medical University

Ju Cao ( $\square$ caoju723@163.com )

Chongqing Medical University

\section{Research article}

Keywords: Thymic stromal lymphopoietin, Systemic Lupus Erythematosus, lupus nephritis, disease activity, cytokine-receptor complex Posted Date: November 3rd, 2021

DOI: https://doi.org/10.21203/rs.3.rs-910614/v1

License: (1) This work is licensed under a Creative Commons Attribution 4.0 International License. Read Full License 


\section{Abstract}

Objective To investigate the association between Thymic stromal lymphopoietin (TSLP) and disease activity in patients with Systemic Lupus Erythematosus (SLE).

Methods In this study, concentrations of serum TSLP in 65 SLE patients, 50 sex and age-matched control subjects were determined by enzyme-linked immunosorbent assay (ELISA).

Results Serum TSLP concentrations in SLE patients were dramatically higher than healthy controls. The levels of serum TSLP displayed a significant increase as compared with healthy controls. More importantly, TSLP levels were significantly correlated with SLE disease activity features such as ESR, CRP, Anti-dsDNA Ab, and SLEDAI-2K. The predictive value of TSLP on high disease activity was superior to those of CRP, ESR, and Anti-dsDNA Ab. A note worthy correlation in our study was observed between the serum TSLP levels and laboratory parameters, particularly serum lipids. Furthermore, serum TSLP levels could be significantly down-regulated after effective integrative treatment.

Conclusion TSLP may serve as a novel sensitive biomarker to assist disease activity assessment and monitor therapeutic effects in active SLE patients.

\section{Introduction}

Systemic Lupus Erythematosus (SLE) is a systematic autoimmune inflammation disease, which figured as auto-antibody directly binds nucleic acids with epitopes, forming immune complex and thus leading to chronic inflammatory and multiple organ damage ${ }^{[1-2]}$.The interactions between numerous inflammatory cells including T/B lymphocytes, macrophages, and neutrophils together with various cytokines and chemokines are considered as the main cause of Systemic inflammation. Especially when immune dysfunction like immune complex formation happens, the SLE may induce immune damage with different pathological types of kidneys, resulting in lupus nephritis $(\mathrm{LN})^{[3]}$

Therefore, declaring the role of inflammatory factors of the host in SLE and LN is able to provide new approaches for SLE diagnosis and cure ${ }^{[4,5]}$. Currently, the treatment strategy towards SLE is early detection and early application of comprehensive treatments such as disease-improving anti-rheumatic drugs (DMARD) or biological agents, together with regular assessment of disease activity till symptoms are relieved or reduced. For patients in the active phase, the identification of critically ill patients is important for modifying treatment plans and improving prognosis ${ }^{[6,7]}$. Therefore, the correct assessment of SLE activity has a great means for rapid diagnosis and accurate treatment.

Thymic stromal lymphopoietin (TSLP) is a potent immunomodulatory cytokine that belongs to the IL-2 cytokine family, initially thought to be a growth and differentiation factor for T and B lymphocytes. The TSLP is mainly expressed by activated pulmonary and intestinal epithelial cells, keratinocytes and fibroblasts, dendritic cells and mast cells are also found able to secret TSLP. Human TSLP is a fourhelix bundle cytokine formed by three pairs of intra-chain disulfide bonds. The coding gene is located on chromosome 5q22.1. There are two main homologous forms of human TSLP, short form (sfTSLP) and long form (IfTSLP). IfTSLP encodes 159 amino acids with a molecular weight of about $14.9 \mathrm{kD}$. It is usually up-regulated during inflammation and exerts a pro-inflammatory effect ${ }^{[9]}$. sfTSLP is mainly composed of 60 amino acids. It is often expressed in a healthy state and exerts a homeostasis effect. However, the expression level of TSLP in the peripheral blood of SLE patients are unclear.

At present, there are laboratory indicators like Erythrocyte Sedimentation Rate (ESR), C-Reactive Protein (CRP), and Anti-dsDNA Antibody are used to assess clinical disease activity, yet these indicators are insufficient in sensitivity and specificity to evaluate active SLE. It has been reported that SLEDAI-2K and renal SLEDAI scores may be related to the severity of SLE ${ }^{[13,14]}$. Therefore, seeking a sensitive and objective biomarker is of great significance for evaluating the disease activity of patients with SLE. Thus, the purpose of this research is to identify the potential relationship between serum TSLP concentration and SLE activity, evaluate whether TSLP could be a biomarker for SLE high activity and efficacy monitoring. Besides, this research also investigates the TSLP production in LN patients, which carries a potential significance to SLE diagnosis and treatment.

\section{Materials And Methods}

\subsection{SLE patients, control subjects and blood samples}


65 Chinese patients with SLE fulfilling American College of Rheumatology (ACR97) and/or Systemic Lupus International Collaborating Clinics (SLICC'12) classification criteria, were recruited at The First Affiliated Hospital of Chongqing Medical University from the years of 2019 to 2021.Diagnosis of SLE was established according to the 1982 revised American Rheumatism Association criteria ${ }^{[15]}$, and the SLE disease activity was measured according to the SLE is ease Activity Index(SLE-DAI) and patients with SLE-DAI score $\geq 5$ were defined active SLE ${ }^{[16,17]}$. The SLE patients were also divided into two groups:35 SLE patients with renal disease(LN group) and 30 SLE patients without renal disease(non-LN group). SLE patients younger than 18 , concurrent infectious diseases, malignant cancer, or other autoimmune diseases were excluded. Data were collected from each patient's standardized electrical medical records. It needs to be pointed out that after effective treatment of 30 patients with moderate and high activity SLE, their clinical characteristics will be reassessed during the subsequent 8-week follow-up. This project adheres to the principles of the Declaration of Helsinki and was approved by the ethics committees of the Clinical Research Ethics Committee of the First Affiliated Hospital of Chongqing Medical University. Informed consent was obtained from all patients.

\subsection{Definition of Lupus nephritis}

Lupus nephritis (LN) refers to systemic lupus erythematosus (SLE) with different pathological types of renal immune damage, accompanied by obvious clinical manifestations of renal damage. Its pathogenesis is related to the formation of immune complexes, immune cells and cytokines. In addition to systemic manifestations of SLE, the main clinical manifestations were hematuria, proteinuria and renal insufficiency. The pathological classification of lupus nephritis is of great value in judging the disease activity and prognosis, and making treatment plan. The treatment plan should be individualized according to the severity of the disease.Renal biopsy-proven LN specimens were evaluated according to the classification criteria defined by the ISN/RPS.Lupus nephritis can be divided into mild and severe according to the degree of renal pathological injury..Renal activity of SLE was assessed by renal SLEDAI score,which consisted of proteinuria, urinarycasts, hematuria and pyuria of the original SLEDAI score ${ }^{[18]}$. Systemic lupus erythematosus (SLE) is a diffuse autoimmune disease characterized by the involvement of multiple organs and multiple systems. The lung is a common organ involved in SLE, with various manifestations, including pleural effusion, interstitial pneumonia, pulmonary hypertension, diffuse alveolar hemorrhage, pulmonary embolism, etc. The overall incidence of lung impairments is relatively high ${ }^{[20,21]}$.Among them, interstitial lung disease (Interstitial lung disease, ILD) is a common comorbidity of SLE, which seriously affects the quality of life and prognosis of patients. Due to the insidious onset of interstitial pneumonia and the lack of specific serum markers, irreversible fibrotic lesions have often occurred at the time of diagnosis Therefore, the serum TSLP levels of 10 SLE-ILD patients were observed in this study.

\subsection{Data collection}

The information of SLE patients covered demographic data,including age,sex,disease duration,clinical manifestations,and laboratory examinations. SLE-related features included rash/ discoidrash, oral ornasal ulcers,alopecia, serositis,arthritis, active nephritis,CNS (central nervous system)lupus, vasculitis, fever $>38^{\circ} \mathrm{C}$,thrombocytopenia, leukopenia, and anemia and were based on the

SLEDAI except anemia ${ }^{[19,20]}$.For instance,anemia,thrombocytopenia and leukopenia were defined as adecrease in the concentration of hemoglobinto $<100 \mathrm{~g} / \mathrm{l}$, in thenumber of platelets to $<100 * 10^{9} / \mathrm{l}$, and in the number of white blood cells to $<3 * 10^{9} /$.

\subsection{Laboratory markers}

The concentrations of serum C3, C4 and C-reactive protein (CRP) were measured by turbidimetric immunoassay on Beckman Immage 800 immunology analyzer. The levels of serum anti-ANA,anti-Sm Ab,anti-dsDNA antibodies were measured by using fluorescenceenzyme immunoassay (Euroummun, USA). The erythrocyte sedimentation (ESR) levels were manually performed via Westergren method.The serum TSLP levels were determined by using enzyme linked immunosorbent assay (ELISA) kits (USA R\&D Systems) according to the manufacturer's protocol.

\subsection{Whole blood assay}

This method was established according to the report previously ${ }^{[21]}$. After a Maximum storage period of $1 \mathrm{~h}$ of collected EDTA blood at room temperature, blood samples were diluted 1:1 with RPMI 1640(Gibco Laboratories, NY, USA), and 1 ml aliquots were dispensed in each well of a 24-well plate (Nalge Nunc International, IL, USA). The blood culture was then stimulated with or without phytohaemagglutinin (PHA, a T-cell mitogen; Sigma, MO, USA) at $5 \mu \mathrm{g} / \mathrm{ml}$ and lipopolysaccharide (LPS, a mitogen of B cells and 
macrophages; Sigma) at $25 \mu \mathrm{g} / \mathrm{ml}$ for $24 \mathrm{~h}$. After incubation, the cell-free supernatant from ex vivo cultures was harvested for subsequent ELISA of TSLP. The absolute number (cells/ $\mu$ l) of leucocytes (CD45+) of each whole blood sample was measured with the Multitest IMK Kit with Trucount Tubes (Becton Dickinson, CA, USA) using the lyse/no-wash method with a four-colour FACS Calibur flow cytometer (Becton Dickinson). To normalize the individual difference in leucocyte number of each whole blood sample,the ex vivo production of TSLP was expressed as $\mathrm{ng} / 10^{6}$ leucocytes.

\subsection{Statistical analysis}

All analyses were performed using the Statistical Package for the Social Sciences (SPSS) statistical software for Windows, version 9.0 (SPSS, IL, USA). The difffferences between two groups were analyzed by Mann-Whitney U test. The relations between TSLP levels and other continuous variables were analyzed by using the Mann-Whitney $U$ test as well. The CD5L levels before or after treatment were compared by using a paired t-test. Furthermore, the SLE patients were divided into three groups based on scores of clinical features $(0,1$ to 2 , and 3 to 8 ). Among three groups, $p$ values were calculated by analysis of variance and were adjusted by use of the Bonferroni correction. $p$ values $<0.05$ were considered significant.

\section{Result}

\subsection{The Demographic and Clinical Characteristics of Study Subjects}

Totally, there were 65 patients with SLE and 30 healthy controls in the current study. Among the 65 SLE patients, 35 had active disease, while 30 had inactive disease. There is no difference between HC (health control) and SLE patients in the aspect of age distribution. The proportion of females $(97.1 \%, \mathrm{p}=0.023)$ was higher in patients with active disease than those with inactive disease $(86.6 \%)$ and $\mathrm{HC}$ (50\%). The proportion of SLE patients (52\%) from rural areas has no obvious difference between that of the control group (50\%). The duration of SLE was 6.5(1.8-8.9) years (median (interquartile range, IQR)) and 8.9 $(3.6,10.6)$ years (median (IQR)) for inactive and active patients, respectively.

There was no significant difference in the count of WBC ( $6.6 \mathrm{vs} 6.1, p=0.071$ ) between the SLE inactive group and the control group, while the percentage of neutrophils ( $32 \%$ vs $65 \%, p=0.013$ ) in the SLE active group was lower than that of the control group, and the percentage of lymphocytes ( $68 \%$ vs $32 \%, p=0.001)$ was significantly higher.

\subsection{TSLP level upregulated in serum of SLE patients}

To investigate the TSLP secretion in SLE patients, TSLP concentrations of 65 serum samples from SLE patients were tested by ELISA. The TSLP level in patients are significantly higher comparing to healthy controls[Median (interquartile range): SLE patients 575 (278.2, 937.6) $\mathrm{pg} / \mathrm{ml}$, healthy control group $59.7(41.78,150.8) \mathrm{pg} / \mathrm{ml}$. (Fig. 1A) To the aspect of gender distribution, the TSLP level in females is obviously higher than in males (Fig. 1C), and the TSLP level of SLE patients decreased slightly with age but did not change significantly (Fig. 1D).

In order to further investigate the production of TSLP in SLE patients, patients were divided into the following three groups according to the presence of comorbidities: SLE-LN group $(n=25)$, SLE-ILD group $(n=10)$, and no serious complications SLE group $(n=30)$.

Compared with the SLE group, the serum ESR, CRP, Anti-dsDNA Ab, C3, SLEDAl, and SLEDAI-2K scores were significantly different in the SLE-ILD group $(P<0.05)$. Importantly, we found that the serum TSLP level of the SLE-LN group (median $=814 \mathrm{pg} / \mathrm{ml}$ ) was significantly higher than that of the SLE-ILD group and SLE group $(p=0.007$ and $0.0001, P<0.01)$, as shown in Fig. $1 \mathrm{~B}$, indicating that it is related to the severity of the disease. In addition, the serum TSLP level of the SLE-ILD group (Medium $=928 \mathrm{pg} / \mathrm{ml}$ ) was also higher than that of the SLE group (median $=575 \mathrm{pg} / \mathrm{ml}, \mathrm{p}=0.002)$ (Fig. 1B). Serum TSLP levels are also significantly different in the mild, moderate, and severe disease groups. (Fig. 1D)

\subsection{Relationship between serum TSLP and SLE activity}

The TSLP levels of SLE patients with different disease activities were evaluated next. The clinical features were shown in Table 1.

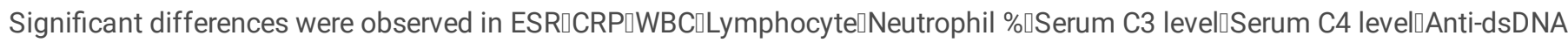
levels $(\mathrm{U} / \mathrm{ml}) \square S L E D A l$ score between different severity groups, while WBC and Serum C4 levels have no obvious differences. As shown in Fig. 2, the median of TSLP was $286.8 \mathrm{pg} / \mathrm{ml}$ and $929.6 \mathrm{pg} / \mathrm{ml}$, respectively, showing an increasing trend from the stable group to the active group. Compared with other groups, the serum TSLP level of the high activity group was significantly increased $(p=0.021$ and 0.0003). And TSLP SLEDAl, $(r=0.448, p=0.002)$, erythrocyte sedimentation rate $(r=0.491, p<0.0001)$, and C-reactive protein $(r=0.621$, 
$\mathrm{p}<0.0001)$ showed a significant positive correlation. Therefore, the TSLP level may be related to SLE disease activity. Potential manifestations of severe lupus, such as hemolytic anemia, pneumonia, or gastrointestinal involvement, are not scored in SLEDAI.

The TSLP levels of SLE patients with different disease activities were evaluated next. The clinical features were shown in Table 1.

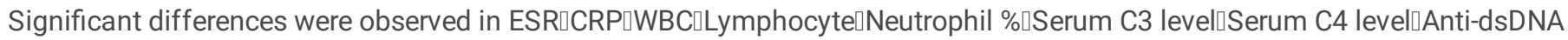
levels $(\mathrm{U} / \mathrm{ml}) \square S L E D A l$ score between different severity groups, while WBC and Serum C4 levels have no obvious differences. As shown in Fig. 2, the median of TSLP was $286.8 \mathrm{pg} / \mathrm{ml}$ and $929.6 \mathrm{pg} / \mathrm{ml}$, respectively, showing an increasing trend from the stable group to the active group. Compared with other groups, the serum TSLP level of the high activity group was significantly increased $(p=0.021$ and 0.0003). And TSLP SLEDAl, $(r=0.448, p=0.002)$, erythrocyte sedimentation rate $(r=0.491, p<0.0001)$, and C-reactive protein $(r=0.621$, $p<0.0001)$ showed a significant positive correlation. Therefore, the TSLP level may be related to SLE disease activity. Potential manifestations of severe lupus, such as hemolytic anemia, pneumonia, or gastrointestinal involvement, are not scored in SLEDAl.

\subsection{Correlation between TSLP level and laboratory indicators in SLE patients}

Serum TSLP levels in SLE patients are significantly correlated with C3, Anti-ds-DNA and SLEDAl levels $(r=-0.7109, p<0.0001 ; r=0.6209$, $p=0.0003 ; r=0.8579, p<0.0001)$, as shown in Fig. $3 A$ and $3 B$. It is particularly noteworthy that TSLP may be involved in the production of multiple antibodies in the pathogenesis of SLE.

\subsection{Effect of comprehensive treatment on TSLP in patients with SLE}

In order to understand the effect of comprehensive treatment on the TSLP level of SLE-like patients, 30 SLE patients with mild, moderate, and high disease activity were included in this study for efficacy evaluation. Among them, the mild patient group took Vitamin $\mathrm{C}$ as a placebo, and the moderate and severe SLE patients The active group took anti-rheumatic drugs, Glucocorticoid and Rituximab, respectively. After 8 weeks of comprehensive treatment, the TSLP level score of each treatment group was significantly lower than that of the placebo group $(P<0.001$, Fig. 3A), and SLEDAI-2K changed significantly before and after treatment $(P<0.0001, F i g .3 B)$. After treatment, $C R P$, anti-Ds-DNA Ab, erythrocyte sedimentation rate and other disease activity indicators were significantly reduced $(P<$ 0.05). ( Table 2.)

\section{6 correlation between serum TSLP and clinical indexes before and after treatment}

As shown in Table 2, the serum level of TSLP in SLE patients was significantly positively correlated with the levels of anti dsDNA, ESR, $\mathrm{CRP}$ and sledai-2k score. There was no significant correlation with $\mathrm{C} 4, \mathrm{WBC}, \mathrm{Hb}$ and PLT. Table 2. The correlation of TSLP and clinical indicators after eight-week integrative medicine treatment.

\subsection{Ex vivo production of TSLP in SLE patients}

Fig 4. shows that Lymphocyte active antigen (PHA) and LPS could signifificantly elevate the release of TSLP from peripheral blood mononuclear cells (PBMC) compared with medium control in healthy controls and SLE patients. Moreover, the percentage increase in ex vivo production of TSLP after incubation with PHA and LPS was signifificantly higher in SLE patients than in control subjects.

\section{Discussion}

In recent years, some studies have focused on new mechanisms affecting the innate and acquired immune systems in different stages of systemic lupus erythematosus ${ }^{[22]}$. Cytokines play an important role in the pathogenesis of inflammation regulation and affect the severity of autoimmune diseases ${ }^{[23]}$. Further analysis showed that thymic stromal lymphopoietin (TSLP) is an IL-7-related cytokine, which has been extensively studied in atopic and rheumatic diseases. The landscape of therapeutic agents that can modulate the bioactivity of TSLP and IL-7 ininflammation, autoimmunity and cancer is clearly very broad in terms of disease coverage and displays a strong focus on biologics. Considering the important role of both TSLP and IL-7 in the pathogenesis of RA simultaneous inhibition of both TSLP and IL-7R signaling in arthritis could serve aplausible the rapeutic rationale in arthritis.

Multiple studies have also shown that in human cells and animal models of Rheumatoid arthritis (RA) and Lupus nephritis (LN), overexpression of TSLP can induce T cell activation and pro-inflammatory cytokine production. Therefore, TSLP-IL17-a receptor axis has great potential for targeted therapy. Another important feature of TSLP is its ability to act as an initiator of allergic inflammation in

Page 5/13 
both human and mouse ${ }^{[24,25]}$. Elevated TSLP levels are found in affected skin and lung from patients of a topic dermatitis or allergic asthma,respectively ${ }^{[27]}$. Consistent with this , mice overexpressing TSLP or administered with recombinant TSLP exhibit severe Th2polarlized inflammation in the sites ${ }^{[28]}$.

However, as far as we know, there is no research report on TSLP and SLE. Here, we provide the first evidence that TSLP can be used as a biomarker for predicting SLE disease activity and monitoring treatment. In this study, we found that patients with systemic lupus erythematosus (SLE), regardless of the stable group, the active group, the lupus nephritis group, and the lupus-related interstitial lung disease group, had significantly higher serum TSLP levels than the healthy control group. It suggests that TSLP may be involved in the pathological process of systemic lupus erythematosus host immune response and provides a new perspective for identifying the disease activity of systemic lupus erythematosus. More importantly, our research results show that according to the SLEDAI-2K score, the TSLP level of patients with moderate and high activity SLE is significantly higher than that of patients with low activity SLE, indicating that the high activity of SLE has higher sensitivity and specificity. Therefore, TSLP can be used as a new and sensitive biomarker for SLE with moderate to high disease activity.

Significantly, lupus nephritis and interstitial lung disease are common comorbidities in patients with SLE, and the higher the cumulative disease activity, the higher the risk of comorbidities ${ }^{[32-34]}$. In our study, we found that the serum TSLP level of the SLE-ILD group was significantly higher than that of the SLE group without comorbidities. This means that high TSLP levels may be related to the severity of the disease, thereby predicting the poor prognosis of active SLE.

Certain biomarkers are more valuable than diagnostics in guiding treatment and prognosis. For example, several biomarkers such as TNF-a, IL-6, IL-33 and MMP-3 are used to evaluate the effect of clinical treatment ${ }^{[35-38]}$. Our current results show that after effective comprehensive treatment, the serum level of TSLP may decrease significantly. In addition, we also focused on the changes in clinical characteristics of SLE patients with moderate and high disease activity before and after treatment and their correlation with TSLP levels. Therefore, TSLP may become a potential marker for monitoring clinical efficacy.

However, some limitations of our research should be considered. On the one hand, there are relatively few samples of lupus-related interstitial lung disease, which need to be further verified in the expanded sample; on the other hand, the number of cases in the biologics treatment group is too small to represent the expression level of TSLP. In our study, most patients have long-term SLE, and disease activity is assessed at the time of admission, which may not represent all chronic inflammation in the entire course of the disease. In the future, a multicenter study based on long-term follow-up is still needed to further evaluate the patient's condition at the time of onset.

In summary, our research results indicate that TSLP may become a new sensitive biomarker for predicting the disease activity of SLE patients and monitoring the effect of treatment, helping to identify patients with higher activity early and to adjust treatment options timely. Therefore, this study provides new ideas for the study of SLE disease activity, but the potential immunopathological role of TSLP in SLE autoimmunity remains to be further studied.

\section{Conclusion}

In summary, we have demonstrated elevated levels of serum TSLP in SLE patients as well as a positive and significant correlation between serum concentration and disease activity. TSLP may serve as a novel sensitive biomarker to assist disease activity assessment and monitor therapeutic effects in active SLE patients.

\section{Abbreviations}

TSLP: Thymic stromal lymphopoietin

SLE: Systemic Lupus Erythematosus

ESR: erythrocyte sedimentation rate

CRP : C-Reactive Protein $\rrbracket$

Anti-dsDNA Ab: Anti-dsDNA Antibody 
SLEDAI-2K: SLE Activity Index 2000

LN: lupus nephritis

DMARD: anti-rheumatic drugs

SLE-DAl: SLE is ease Activity Index

ILD: Interstitial lung disease

CNS: central nervous system

ELISA: enzyme linked immunosorbent assay

HC: health control

SLE-LN: Systemic Lupus Erythematosus lupus nephritis

SLE-ILD: Systemic Lupus Erythematosus lupus Interstitial lung disease

PBMC: peripheral blood mononuclear cells

PHA: Lymphocyte active antigen

LPS: lipopolysaccharide

\section{Declarations}

\section{Ethical Approval and Consent to participate}

This project adheres to the principles of the Declaration of Helsinki and was approved by the ethics committees of the Clinical Research Ethics Committee of the First Affiliated Hospital of Chongqing Medical University.

\section{Consent for publication}

The author agrees to publication in the Journal.

\section{Availability of supporting data}

The data sets supporting the results of this article are included within the article and its additional files.

\section{Competing interests}

All authors declare no conflicts of interest.

\section{Funding}

5.1 Chongqing Education Committee Science Foundation (no. KJQN201900417).

5.2 Research Foundation Natural Science Foundation of China (no. 81901582)

\section{Authors' contributions}

Xiaofei Lai analyzed data, and wrote the paper.Huiqing Yang and Hao Ding performed research, Ju Cao designed research.

\section{Acknowledgements}

The authors gratefully acknowledge support from:

1. Chongqing Education Committee Science Foundation (no. KJQN201900417). 


\section{References}

1. Ramani K, Biswas PS. Interleukin 17 signaling drives Type I interferon induced proliferative crescentic glomerulonephritis in lupusprone mice. Clin Immunol 2016; 162:31-6

2. La Paglia GMC, Leone MC, Lepri G, Vagelli R, Valentini E, Alunno A, Tani C. One year in review 2017: systemic lupus erythematosus. Clin Exp Rheumatol 2017; 35:551-61 『

3. It has been shown that the interleukin (IL)-17/interferon (IFN) type 1 interplay is crucial in the recruitment of immune cells into the kidney and in the formation of the inflammatory infiltrate in lupus nephritis (LN): indeed, lupus-prone IL-17A receptor (IL-17RA) deficient mice develop an IFN type I-dependent LN milder than control mice.

4. Najafi CC, Korbet SM, Lewis EJ, et al. Significance of histological patterns of glomerular injury upon long-term prognosis in severe lupus glomerulonephritis. Kidney Int 2001; 59: 2156-2163.

5. Bettelli E, Oukka M, Kuchroo VK. Th-17 cells in the circle of immunity and autoimmunity. Nat Immunol 2007; 8: 345-350.

6. Xing Q, Wang B, Su H, et al. Elevated Th17 cells are accompanied by FoxP3p Treg cells decrease in patients with lupus nephritis. Rheumatol Int 2012; 32: 949-958. Epub ahead of print 18 January 2011.

7. Kang HK, Liu M, Datta SK. Low-dose peptide tolerance therapy of lupus generates plasmacytoid dendritic cells that cause expansion of autoantigen-specific regulatory T cells and contraction of inflammatory Th17 cells. J Immunol 2007; 178: 78497858.

8. Park JH, Jeong DY, Peyrin-Biroulet L, Eisenhut M, Shin J II. Insight into the role of TSLP in inflflammatory bowel diseases. Autoimmunity Rev. (2017) 16:55-63. doi: 10.1016/j.autrev.2016.09.014

9. El-Ghareeb MI, Helmy A, Al Kazzaz S, Samir H. Serum TSLP is a potential biomarker of psoriasis vulgaris activity. Psoriasis Targets Ther. (2019) 9:59- 63. doi: 10.2147/PTT.S212774

10. Takahashi N, Sugaya M, Suga H, Oka T, Kawaguchi M, Miyagaki T, et al. Thymic stromal chemokine TSLP acts through Th2 cytokine production to induce cutaneous T-cell lymphoma. Cancer Res. (2016) 76:6241- 52. doi: 10.1158/0008-5472.CAN-16-0992.

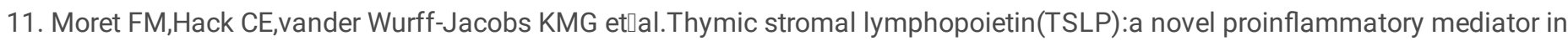
rheumatoid arthritis that potently activates $\operatorname{CD} 1 \mathrm{c}(+)$ myeloid dendritic cellstoattract and stimulate Tcells.Arthritis Rheum 2014;66:117684.49

12. KoyamaK, OzawaT, HatsushikaK et.al. A possible role for TSLP in inflammatory arthritis.BiochemBiophysResCommun2007;357:99104.

13. Furie RA, Petri MA, Wallace DJ, et al. Novel evidence-based systemic lupus erythematosus responder index. Arthritis Rheum 2009;61:1143-51.

14. Diogo Jesus, Ana Matos, Carla Henriques et.al.Derivation and validation of the SLE Disease Activity Score (SLE-DAS): a new SLE continuous measure with high sensitivity for changes in disease activity. Ann Rheum Dis: first published as 10.1136/annrheumdis2018-214502 on 9 January 2019.

15. Tamirou F, Arnaud L, Talarico R, Scire CA, Alexander T, Amoura Z, et al. Systemic lupus erythematosus: state of the art on clinical practice guidelines. RMD Open. (2018) 4:e000793. doi: 10.1136/rmdopen-2018-000793

16. Petri M, Orbai AM, Alarcón GS, et al. Derivation and validation of the systemic lupus international collaborating clinics classification criteria for systemic lupus erythematosus. Arthritis Rheum 2012;64:2677-86.

17. Inês $L$, Rodrigues $M$, Jesus $D$, et al. Risk of damage and mortality in SLE patients fulfilling the ACR or only the SLICC classification criteria. A 10-year, inception cohort study. Lupus 2018;27:556-63.

18. WeeningJJ,D Agati VD, SchwartzMM, etal. The classification of glomerulonephritis in systemic lupus erythematosus revised. J AmSoc Nephrol 2004;15:241-250.

19. Khamashta M, Merrill JT, Werth VP, et al. Sifalimumab, an anti-interferon-a monoclonal antibody, in moderate to severe systemic lupus erythematosus: a randomised, double-blind, placebo-controlled study. Ann Rheum Dis 2016;75:1909-16.

20. Franklyn K, Lau CS, Navarra SV, et al. Definition and initial validation of a Lupus Low Disease Activity State (LLDAS). Ann Rheum Dis 2016;75:1615-21. 
21. C.K. Wong, L.C. Lit, L.S. Tam, E.K. Li, C.W. Lam, Aberrant production of soluble costimulatory molecules CTLA-4, CD28, CD80 and CD86 in patients with systemic lupus erythematosus, Rheumatology (Oxford) 44 (2005) 989-994.

22. Furie R, Khamashta M, Merrill JT, et al. Anifrolumab, an anti-Interferon-a receptor monoclonal antibody, in moderate-to-severe systemic lupus erythematosus. Arthritis Rheumatol 2017;69:376-86.

23. Doria A, Gatto M, Zen M, et al. Optimizing outcome in SLE: treating-to-target and definition of treatment goals. Autoimmun Rev 2014;13:770-7.

24. Zen $M$, laccarino $L$, Gatto $M$, et al. Lupus low disease activity state is associated with a decrease in damage progression in caucasian patients with SLE, but overlaps with remission. Ann Rheum Dis 2018;77:104-10.

25. Ziegler,S.F.andLiu,Y.J.2006.ThymicstromallymphopoietininnormalandpathogenicTcelldevelopmentandfunction.Nat.Immunol.7:709.

26. Liu,Y.J., Soumelis,V., Watanabe,N.etal.2007. TSLP: anepithelial cell cytokine that regulates T cell differentiation by conditioning dendriticcellmaturation. Annu. Rev. Immunol. 25:193.

27. Ziegler,S.F. 2010. The role of thymic stromallymphopoietin (TSLP) inallergic disorders. Curr. Opin. Immunol. 22:795.20 Soumelis,V., Reche,P.A., Kanzler,H.etal.2002. Humanepithelialcell striggerdendritic cell mediated allergic inflammation by producing TSLP. Nat. Immunol. 3:673

28. Connor,B., Ratoff,J. etal.Thymicstromal lymphopoietin expressionis increased in asthmatic airways and correlates with expression of Th2-attracting chemokines anddiseaseseverity.J.Immunol.174:8183.22

29. Astrakhan, A., Omori, M., Nguyen, T. et al. 2007. Local increase in thymic stromal lymphopoietin induces systemic alterations in B cell development. Nat. Immunol. 8:522.

30. Strasser, A., Whittingham, S., Vaux, D. L. et al. 1991. Enforced BCL2 expression in B-lymphoid cells prolongs antibody responses and elicits autoimmune disease. Proc. Natl Acad. Sci. USA 88:8661.

31. Jessica McHugh ,Newly defined pro-inflammatory DC subset expanded in SLE.Nat Rev Rheumatol. 2019 Nov;15(11):637.doi: 10.1038/s41584-019-0311-x.

32. Hongseok Yoo 1, Takuya Hino, Joungho Han etal. Connective tissue disease-related interstitial lung disease (CTD-ILD) and interstitial lung abnormality (ILA): Evolving concept of CT findings, pathology and management .Eur J Radiol Open. 2020 Dec 16;8:100311. doi: 10.1016/j.ejro.2020.100311. eCollection 2021.

33. Jennifer R Hannah 1, David P D'Cruz, Pulmonary Complications of Systemic Lupus Erythematosus Affiliations expand Semin Respir Crit Care Med. 2019 Apr;40(2):227-234. doi: 10.1055/s-0039-1685537. Epub 2019 May 28.

34. Moret FM, Badot V, Lauwerys BR, van Roon JA. Intraarticular soluble interleukin-7 [corrected] receptor levels are increased in patients with rheumatoid arthritis and correlate with local mediators of inflammation: comment on the article by Pickens et al [letter] [published erratum appears in Arthritis Rheum 2012;64: 1302]. Arthritis Rheum 2012;64:594-5.

35. Tanaka J, Watanabe N, Kido M, Saga K, Akamatsu T, Nishio A, et al. Human TSLP and TLR3 ligands promote differentiation of Th17 cells with a central memory phenotype under Th2-polarizing conditions. Clin Exp Allergy 2009;39:89-100.

36. Verstraete K, Peelman F, Braun H, et al. Structure and antagonism of the receptor complex mediated by human TSLP in allergy and asthma. Nat Commun. 2017;8:14937.

37. Park JH, Jeong DY, Peyrin-Biroulet L, et al. Insight into the role of TSLP in inflammatory bowel diseases. Autoimmun Rev. 2017;16(1):55-63.

38. Varricchi G, Pecoraro A, Marone G, et al. Thymic stromal lymphopoietin isoforms, inflammatory disorders, and cancer. Front Immunol. 2018;9:1595.

\section{Tables}

Due to technical limitations, table PDF is only available as a download in the Supplemental Files section.

\section{Figures}



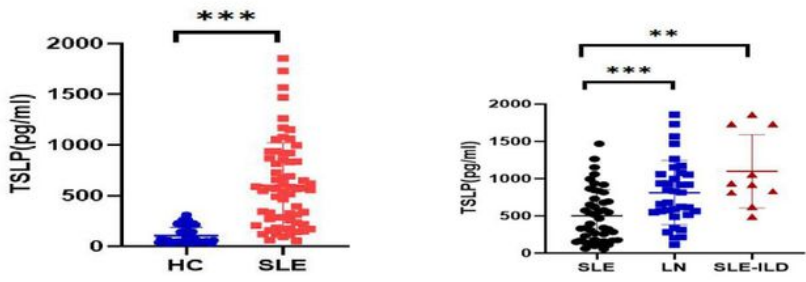

A (HC group=50,. SLE group=65)

B (SLE group-46, LN group-35, SLE-ILD group=10)
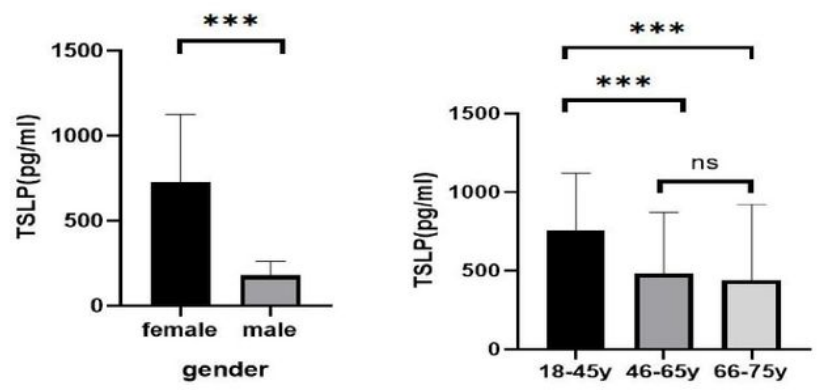

$C($ female group $=50$, male group $=15$ )

D (18-45y group $=30,46-65$ y group $=22,66-75$ y group $=13$ )
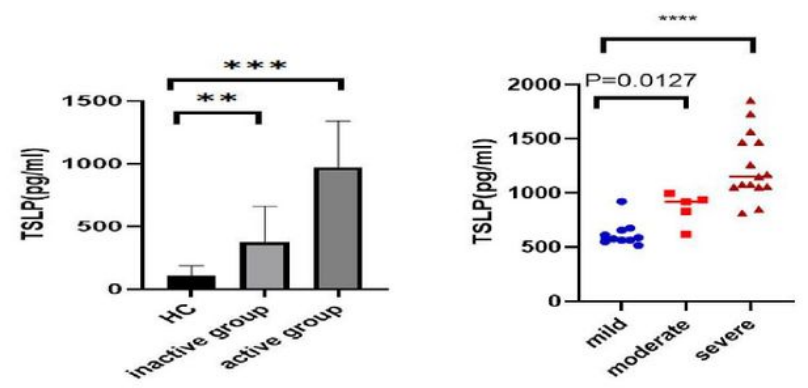

E (HC group=50, inactive group-20,active group-30,

$F($ mild goup $=10$, moderate group $=5$, severe group $=15$ )

\section{Figure 1}

Serum TSLP concentrations were elevated in SLE patients. A: Determination of TSLP concentrations in patients with SLE and the controls. B: Scatter-plots of serum TSLP levels in SLE patiens with different comorbidities. C: Concentrations of serum TSLP in different gender groups. $p<0.05$ as statistically significant.. D: Concentrations of serum TSLP in different age groups. $p<0.05$ as statistically significant E:TSLP levels of SLE patients at different disease activity status group $\left({ }^{\star} p<0.05 ; * \star, p<0.01 ; \star \star \star p<0.001\right) F$ :TSLP levels of SLE patients at different disease extenty status group ( $\left.{ }^{*} p<0.05 ; * \star, p<0.01 ; * \star \star p<0.001\right)$ 


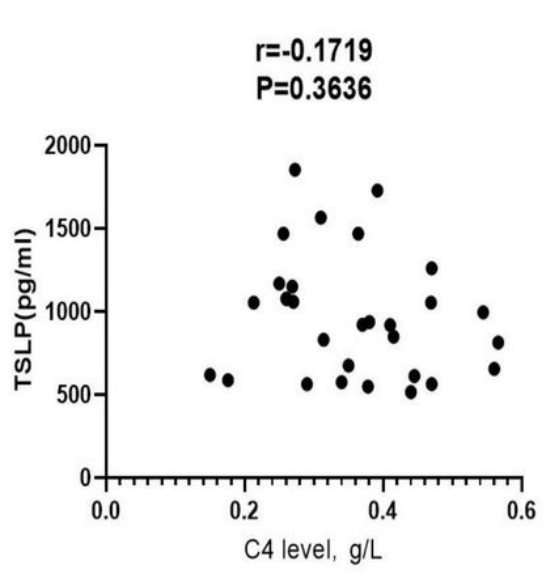

A

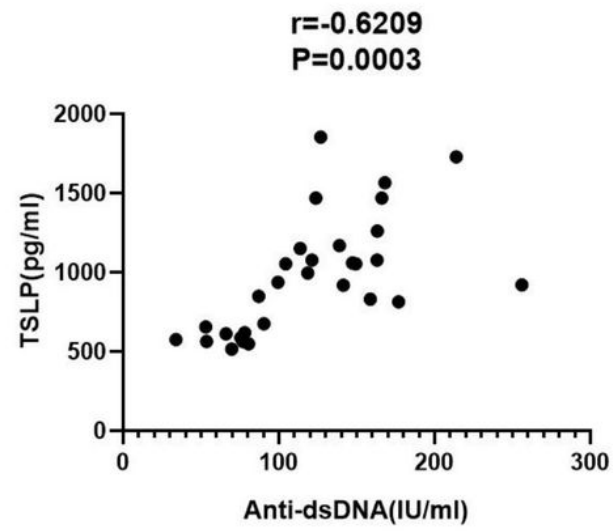

C $r=-0.7109$

$P<0.0001$

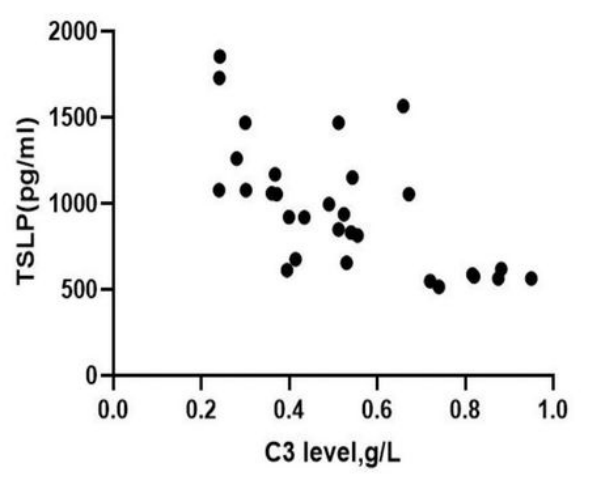

B

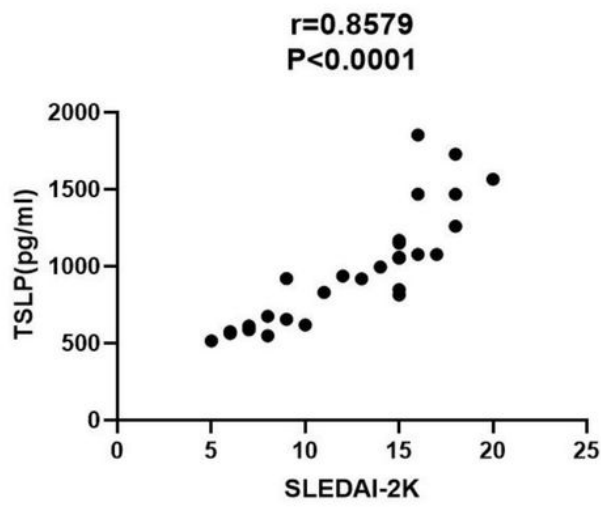

$\mathrm{D}$

\section{Figure 2}

The role of AIM in reflecting disease activity in SLE. A: TSLP levels of SLE patients at different disease activity status based on SLEDAI पESR score. Each symbol represents an individual subject, and horizontal bars represent median values. The difference between groups was determined by non-paremetric Mann-Whitney rank sum test ( $\left.{ }^{*} p<0.05 ;{ }^{* *}, p<0.01\right)$ B-D: Correlation of serum TSLP concentretion with SLE disease activity paremeters including C3ロC4ロAnti-dsDNA Ab and SLEDAI-2K. 

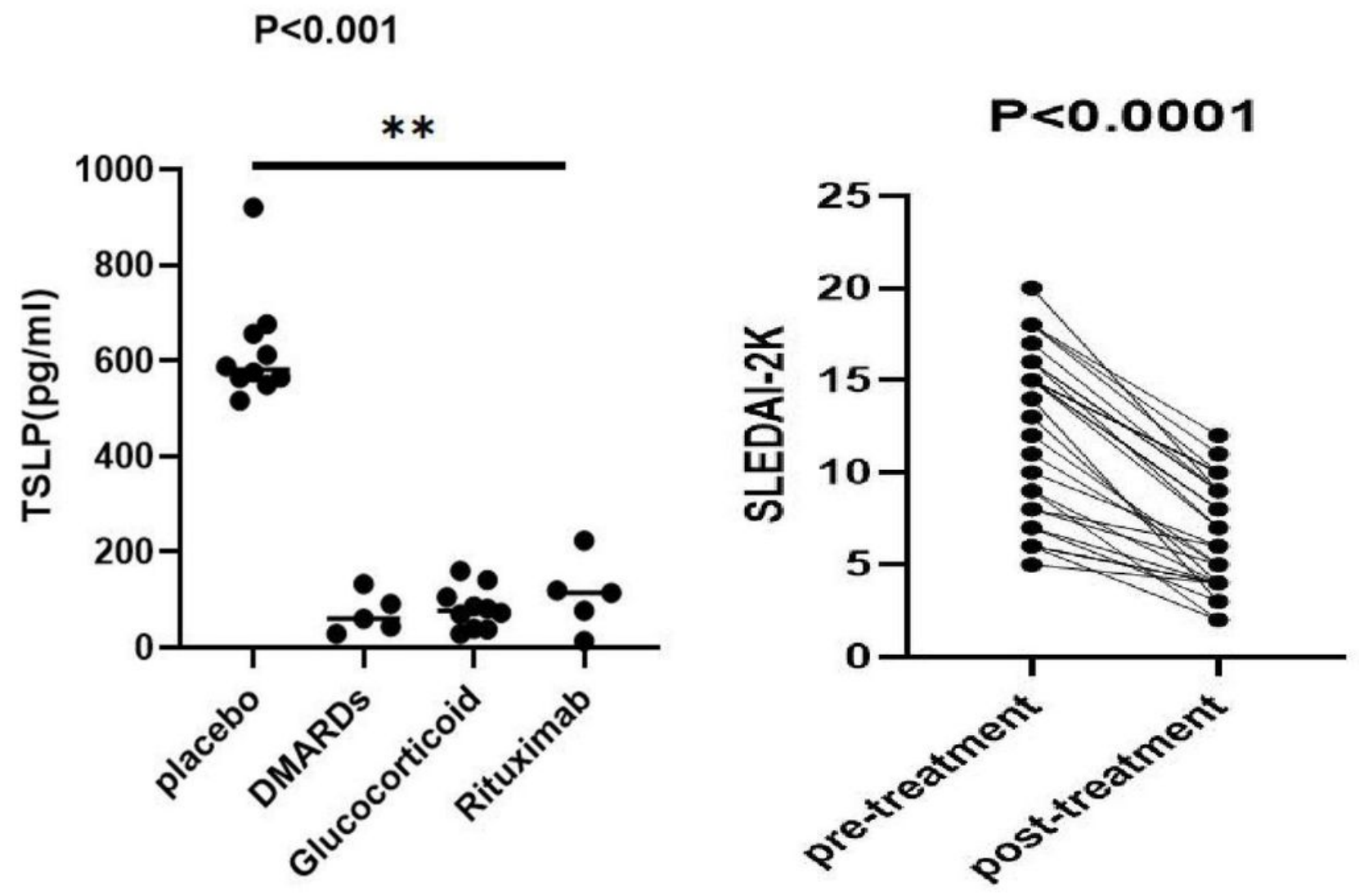

A B

Figure 3

Effect of treatment with integrative medicine on the production of TSL 


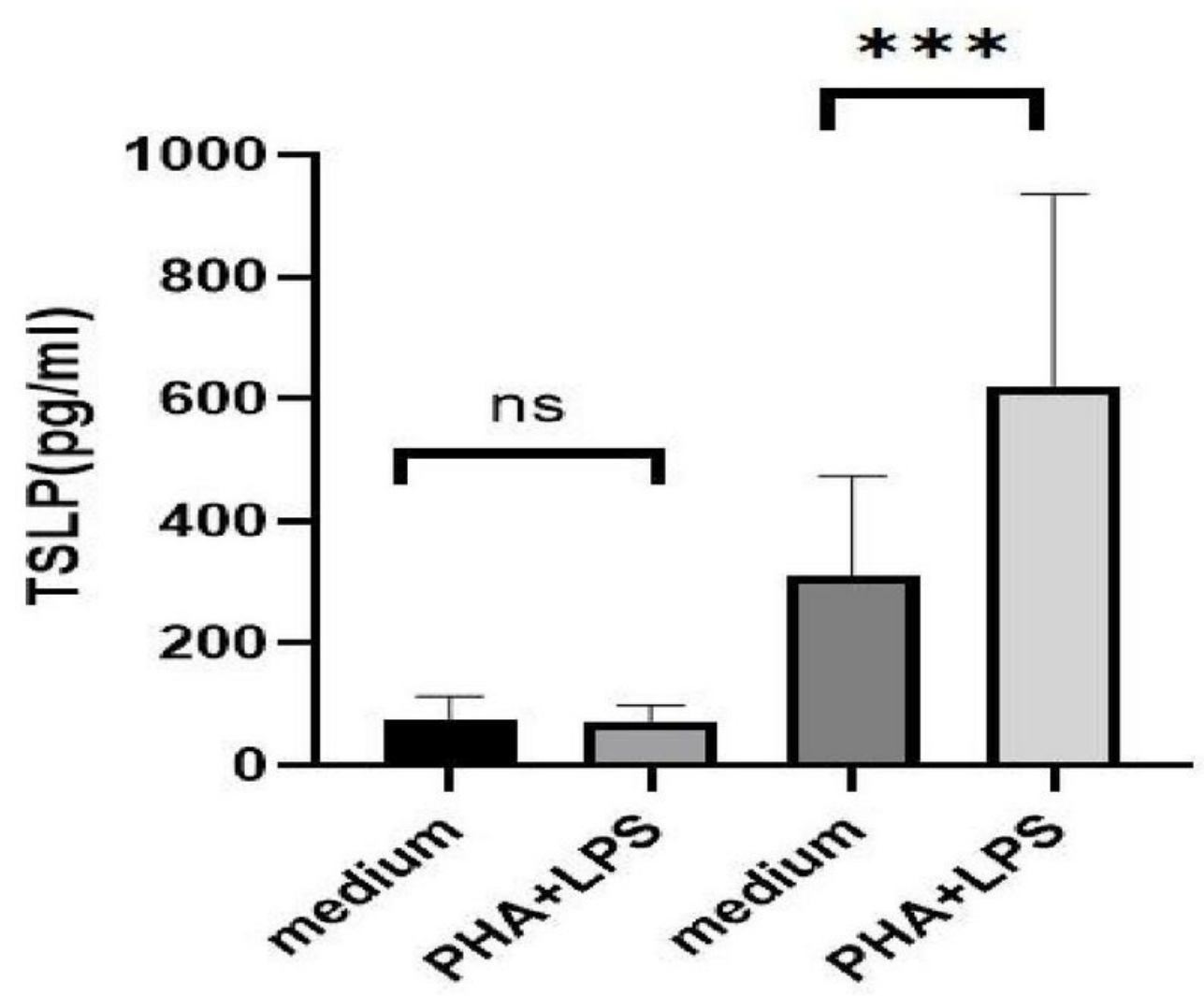

\section{Healthy contor}

\section{Lupus nephritis}

Figure 4

Ex vivo production of TSLP upon mitogen activation of PBMC of $12 \mathrm{LN}$ patients and 12 healthy controls. The culture supernatant for TSLP measurement by ELISA was derived from whole blood cultured with medium in the absence or presence of PHA $(5 \mu \mathrm{g} / \mathrm{ml}) \mathrm{and}$ LPS $(25 \mu \mathrm{g} / \mathrm{ml})$ for $24 \mathrm{~h}$. Results are presented Scatter-plots. The Mann-Whitney U test was used to assess differences in TSLP concentrations between different groups.

\section{Supplementary Files}

This is a list of supplementary files associated with this preprint. Click to download.

- table.pdf 